\title{
The Primate Thalamus Is a Key Target for Brain Dopamine
}

\author{
Miguel Ángel Sánchez-González, Miguel Ángel García-Cabezas, Beatriz Rico, and Carmen Cavada \\ Departamento de Anatomía, Histología y Neurociencia, Facultad de Medicina, Universidad Autónoma de Madrid, 28029 Madrid, Spain
}

The thalamus relays information to the cerebral cortex from subcortical centers or other cortices; in addition, it projects to the striatum and amygdala. The thalamic relay function is subject to modulation, so the flow of information to the target regions may change depending on behavioral demands. Modulation of thalamic relay by dopamine is not currently acknowledged, perhaps because dopamine innervation is reportedly scant in the rodent thalamus. We show that dopaminergic axons profusely target the human and macaque monkey thalamus using immunolabeling with three markers of the dopaminergic phenotype (tyrosine hydroxylase, dopamine, and the dopamine transporter). The dopamine innervation is especially prominent in specific association, limbic, and motor thalamic nuclei, where the densities of dopaminergic axons are as high as or higher than in the cortical area with the densest dopamine innervation. We also identified the dopaminergic neurons projecting to the macaque thalamus using retrograde tract-tracing combined with immunohistochemistry. The origin of thalamic dopamine is multiple, and thus more complex, than in any other dopaminergic system defined to date: dopaminergic neurons of the hypothalamus, periaqueductal gray matter, ventral mesencephalon, and the lateral parabrachial nucleus project bilaterally to the monkey thalamus. We propose a novel dopaminergic system that targets the primate thalamus and is independent from the previously defined nigrostriatal, mesocortical, and mesolimbic dopaminergic systems. Investigating this "thalamic dopaminergic system" should further our understanding of higher brain functions and conditions such as Parkinson's disease, schizophrenia, and drug addiction.

Key words: dopamine; dopamine transporter; dopaminergic systems; thalamus; human; monkey

\section{Introduction}

The thalamus transfers sensory information from the periphery to the cortex through first-order relay nuclei. In addition, it is a nodal point in corticocortical communication through higherorder relay or association nuclei (Sherman and Guillery, 1996). These functions are subject to extensive modulation, and thus the transfer of information through the thalamus depends on the state of wakefulness of the subject and on attentional or behavioral demands (McCormick, 1992; Sherman and Guillery, 1996; Steriade et al., 1997; Guillery and Sherman, 2002). In fact, the vast majority of synapses in the thalamus are from modulatory afferents. The other main targets of thalamic efferents are the striatum, the nucleus accumbens, and the amygdala (Steriade et al., 1997).

Dopamine is an important transmitter molecule in the brain. By modulating neuronal excitability and synaptic plasticity

Received March 11, 2005; revised April 29, 2005; accepted May 16, 2005.

This work was supported by Ministries of Education and Science of Spain (Grants PB 98-0064 and BFI200200513). B.R. and M.A.S.-G. were recipients of fellowships from the Ministry of Education and Science (B.R. and M.A.S.-G.) and from the Community of Madrid (M.A.S.-G.). We are grateful to the Pathology Department of the Hospital Universitario La Paz of Madrid, in particular to Drs. Manuel Gutiérrez and Carmen Morales, for their support and for giving us access to human brain tissue from their necropsy procedures. We appreciate the helpful comments about this manuscript by Drs. Carlos Avendaño, Francisco Clascá, and Wolfram Schultz. Gemma De la Fuente, Piedad Romero Ruiz, and Rosa Sánchez Lozano provided expert technical assistance during surgeries and tissue processing.

Correspondence should be addressed to Dr. Carmen Cavada, Departamento de Anatomía, Histología y Neurociencia, Facultad de Medicina, Universidad Autónoma de Madrid, C/ Arzobispo Morcillo s/n, 28029 Madrid, Spain. E-mail: carmen.cavada@uam.es.

B. Rico's present address: Instituto de Neurociencias de Alicante, Universidad Miguel Hernández-Consejo Superior de Investigaciones Científicas, 03550 Sant Joan d'Alacant, Alicante, Spain.

D01:10.1523/JNEUROSCI.0968-05.2005

Copyright $\odot 2005$ Society for Neuroscience $\quad$ 0270-6474/05/256076-08\$15.00/0
(Nicola et al., 2000; Bissiere et al., 2003), dopamine is essential for motor control (Agid, 1991), working memory (Brozoski et al., 1979), sensorimotor learning (Aosaki et al., 1994), and rewardrelated learning and motivation (Schultz, 2002). These functions depend on the dopamine innervation of the striatum, cerebral cortex, nucleus accumbens, and amygdala. This innervation originates in dopaminergic neurons of the ventral mesencephalon and makes up the nigrostriatal, mesocortical, and mesolimbic dopaminergic systems (Lewis and Sesack, 1997).

The presence of dopamine in the thalamus of primates was reported a long time ago using biochemical tools (Brown et al., 1979; Goldman-Rakic and Brown, 1981). More recently, axons that were immunoreactive (ir) for the dopamine transporter (DAT) have been reported in the monkey mediodorsal thalamic nucleus (MD) (Melchitzky and Lewis, 2001). Despite these reports, the notion of a significant and widespread dopamine innervation of the primate thalamus is not currently recognized (Sherman and Guillery, 1996, 2004; Steriade et al., 1997), probably because the dopamine innervation of the rodent thalamus is reportedly scant (Groenewegen, 1988; Papadopoulos and Parnavelas, 1990). However, thalamic abnormalities related to dopamine and its receptors have been found in the thalamus of schizophrenic patients (Oke and Adams, 1987; Talvik et al., 2003; Yasuno et al., 2004), and thalamic alterations exist in Parkinson's disease (Rub et al., 2002), a disorder in which dopamine plays a major role.

The present study examines the presence of dopamine in the thalamus of adult human and macaque monkey brains using antibodies against tyrosine hydroxylase (TH; one of the enzymes in the dopamine synthesis pathway), dopamine, and DAT. In 
addition, we have traced the origin of the thalamic dopaminergic axons in the brain of macaque monkeys using retrograde tracttracing combined with immunohistochemistry.

\section{Materials and Methods}

Two human brains were used in this study. They were obtained from two male patients aged 58 and 67 years with no clinical history of any neurological or psychiatric disorder. Brains were removed in routine necropsies performed by the Pathology Department of the Hospital Universitario $\mathrm{La} \mathrm{Paz}$ of Madrid, in accordance with Spanish law on clinical necropsies [Boletín Oficial del Estado (BOE) of June 27, 1980 and BOE of September 11, 1982] and with the approval of the Ethics Committee on Clinical Research of the hospital. Postmortem delays were $2 \mathrm{~h} 45 \mathrm{~min}$ and $3 \mathrm{~h} 30 \mathrm{~min}$. After removal, the brains were fixed by vascular perfusion through both internal carotid arteries and the basilar artery with $3 \mathrm{~L}$ of a saline solution at room temperature, followed by $10 \mathrm{~L}$ of $4 \%$ neutral paraformaldehyde in phosphate buffer $(\mathrm{PB} ; 0.1 \mathrm{M}, \mathrm{pH} 7.4)$ at $4^{\circ} \mathrm{C}$. The intravascular perfusion provides better fixation and preservation of antigenicity than the traditional immersion method alone. The brains were blocked on the stereotaxic coronal plane following the Talairach and Tournoux criteria (Talairach and Tournoux, 1988). These brain blocks, 1 -cm-thick, were postfixed subsequently in $4 \%$ paraformaldehyde for 24-48 $\mathrm{h}$ and then cryoprotected in 30\% phosphate-buffered sucrose.

Eleven adult macaque monkeys (Macaca nemestrina and Macaca mulatta), weighing $4.5-8.5 \mathrm{~kg}$, were used. The data shown come from seven monkeys that gave optimal results. Appropriate measures were taken to minimize animal pain or discomfort, in accordance with European and Spanish guidelines (86/609/EEC and 2003/65/EC European Council Directives; and BOE of March 18, 1988). The monkey experiments were approved by the Bioethics Committee of the Universidad Autónoma de Madrid.

Seven monkeys, used for immunolabeling of dopaminergic axons in the thalamus, were experimentally naive when perfused. Four monkeys were used to elucidate the origin of thalamic dopamine. We stereotaxically injected the retrograde tracer cholera toxin b-subunit conjugated to colloidal gold (CTb-Au; List Biologicals, Campbell, CA) in the following thalamic regions: the midline nuclei (case M40; $10 \mu \mathrm{l}$ ), ventral anterior nucleus (VA; case M39; $5 \mu \mathrm{l}$ ), MD (case M35; $10 \mu \mathrm{l}$ ), and lateral posterior $(\mathrm{LP})$ and ventral lateral (VL) nuclei (case M41; $6 \mu \mathrm{l}$ ) (see Fig. $\left.3 A-D^{\prime}, M\right)$. These nuclei were selected for tract-tracing, because they hold high densities of axons immunoreactive for dopamine markers in the monkey and human thalamus. After a 2 week survival time, these monkeys were perfused.

All animals but one (see below) were anesthetized deeply with sodium pentobarbital and perfused through the ascending aorta with saline, followed by $4 \%$ paraformaldehyde in $\mathrm{PB}$ and a series of $\mathrm{PB}$ sucrose solutions (5-20\%). The brains were stereotaxically blocked on the coronal plane, removed, and cryoprotected in a $30 \%$ sucrose-buffered solution. The monkey used for dopamine immunohistochemistry was perfused with $1 \%$ sodium metabisulfite (MBS; S1516; Sigma, St. Louis, MO) in cacodylate buffer $(0.05 \mathrm{M}, \mathrm{pH} 6)$ followed by the fixative: $1 \% \mathrm{MBS}$ and $5 \%$ glutaraldehyde diluted in $0.1 \mathrm{~m}$ cacodylate buffer, $\mathrm{pH}$ 7.4. After blocking, this brain was immersed in the same fixative for $2 \mathrm{~h}$ with gentle agitation at room temperature, and the blocks were then cryoprotected as above.

The brain blocks from the human and monkey brains were cut on a freezing microtome in coronal sections of $40 \mu \mathrm{m}$ (monkey) and $50 \mu \mathrm{m}$ (human) thickness. Adjacent series of sections were processed to reveal the cytoarchitecture, myeloarchitecture, and chemoarchitecture of the thalamus using cresyl violet staining, silver myelin staining (Gallyas, 1979), and acetylcholinesterase histochemistry, respectively. The acetylcholinesterase activity, which is particularly useful for identifying the thalamic nuclei, was revealed using the protocol described by Cavada et al. (1995).

Immunohistochemical and tracer development procedures. The immunohistochemical detection of dopamine was performed only in monkey tissue, because dopamine is rapidly degraded and can only be preserved with in vivo intravascular perfusion using glutaraldehyde containing sodium metabisulfite. The immunohistochemical detection of DAT was performed using a modified antigen retrieval protocol (Ciliax et al., 1999) consisting of microwave heating of the tissue in sodium citrate buffer, $\mathrm{pH}$ 6, for 15-20 min before primary antibody incubation. This processing greatly enhances the immunoreaction and was critical for revealing DAT in human tissue.

The following primary antibodies were used: mouse monoclonal anti-TH (MAB318; 1:200-1:400; Chemicon, Temecula, CA), rat polyclonal anti-dopamine (NT 104; 1:200-1:250; Eugene Tech, Ridgefield Park, NJ), and rat monoclonal anti-DAT (MAB369; 1:1000; Chemicon). The secondary antibodies used and development were as follows: (1) in the $\mathrm{TH}$ experiments, rabbit anti-mouse secondary antibody (AB240; 1:30; Chemicon) was used, followed by incubation in mouse peroxidaseantiperoxidase (PAP) (PAP14; 1:600; Chemicon), development, and intensification (see below); (2) in the dopamine experiments, rabbit antirat secondary antibody (R3756; 1:200; Sigma) was used, followed by incubation in rat PAP (PAP20; 1:500; Chemicon), development, and intensification (see below); (3) in the DAT experiments, biotinylated rabbit anti-rat secondary antibody (AP164B; 1:400; Chemicon) was used, followed by incubation in avidin-biotin peroxidase complex (ABC Elite; Vector Laboratories, Burlingame, CA) and development (see below).

In human tissue, both $\mathrm{TH}$ and DAT were revealed using the sensitive glucose oxidase-diaminobenzidine (DAB)-nickel method (Shu et al., 1988). Two procedures were used to reveal peroxidase activity in the monkey $\mathrm{TH}$ and dopamine immunohistochemical experiments: a standard DAB protocol $\left(0.05 \% \mathrm{DAB}\right.$ and $\left.0.0015 \% \mathrm{H}_{2} \mathrm{O}_{2}\right)$ and the glucose oxidase-DAB-nickel method. Most sections developed with the standard DAB protocol were mounted and then intensified using a silvergold protocol as described previously (Rico and Cavada, 1998). Some sections were processed following a double-bridging procedure by returning them to the secondary antiserum solution for $1 \mathrm{~h}$ after the incubation in PAP and then again to the PAP complex for 90 min. After this, the sections were developed, mounted, and intensified by immersion in aqueous solutions of $0.005 \%$ osmium tetroxide and $0.05 \%$ thiocarbohydrazide (Alonso and Amaral, 1995). DAT was revealed in monkey tissue using a standard DAB protocol $\left(0.05 \% \mathrm{DAB}\right.$ and $\left.0.003 \% \mathrm{H}_{2} \mathrm{O}_{2}\right)$.

For the detection of the dopaminergic neurons projecting to the thalamus, double-labeling experiments for $\mathrm{CTb}-\mathrm{Au} / \mathrm{TH}, \mathrm{CTb}-\mathrm{Au} / \mathrm{DAT}$, and $\mathrm{CTb}$-Au/dopamine- $\beta$-hydroxylase $(\mathrm{D} \beta \mathrm{H})$ were performed in the monkeys that had received stereotaxic injections of CTb-Au. For the development of CTb-Au, we used a silver enhancement protocol (IntenSE BL silver enhancement kit; RPN492; Amersham Biosciences, Little Chalfont, Buckinhamshire, UK), followed by standard immunohistochemical detection of TH (MAB5280; 1:500; Chemicon), DAT (MAB369; 1:1000; Chemicon), or D $\beta \mathrm{H}$ (CA-301; 1:1000; Protos Biotech Corporation, New York, NY). CTb-Au/D $\beta \mathrm{H}$ double-labeling was performed in sections from the caudal mesencephalon and pons to distinguish between the noradrenergic and the dopaminergic neurons that projected to the thalamus. Both neuronal phenotypes are only present together in the caudal mesencephalon and pons in the same sections. At more rostral brain levels, only dopaminergic cell groups are present, and thus only $\mathrm{CTb}$ $\mathrm{Au} / \mathrm{TH}$ and CTb-Au/DAT were used through the mesencephalon, diencephalon, and telencephalon to reveal the dopaminergic neurons that project to the thalamus (see Table 1 for classification of dopaminergic cell groups).

Control experiments in which the primary antibodies were suppressed from the protocol were run in all immunohistochemical experiments.

All sections were dehydrated in a series of ethanol at increasing concentrations, defatted in xylene, and coverslipped.

Data analysis. The TH-, dopamine-, and DAT-ir axons of selected sections containing the thalamus were drawn using a computerized analysis system (Neurolucida; MicroBrightField, Colchester, VT) attached to a Zeiss (Oberkochen, Germany) Axioskop microscope. These drawings were made on the computer screen using the $40 \times$ objective. The CTb-Au injection sites and the $\mathrm{CTb}-\mathrm{Au} / \mathrm{TH}$ and $\mathrm{CTb}-\mathrm{Au} / \mathrm{D} \beta \mathrm{H}$ double-labeled neurons were drawn with Neurolucida in $1 / 10$ sections using the $10 \times$ and $4 \times$ objectives for the injections and the $20 \times$ objective for the double-labeled neurons.

The numbers of $\mathrm{CTb}-\mathrm{Au} / \mathrm{TH}$ double-labeled neurons were counted. $\mathrm{The} \mathrm{CTb}-\mathrm{Au} / \mathrm{TH}$ double-labeled neurons were chosen for counting, be- 


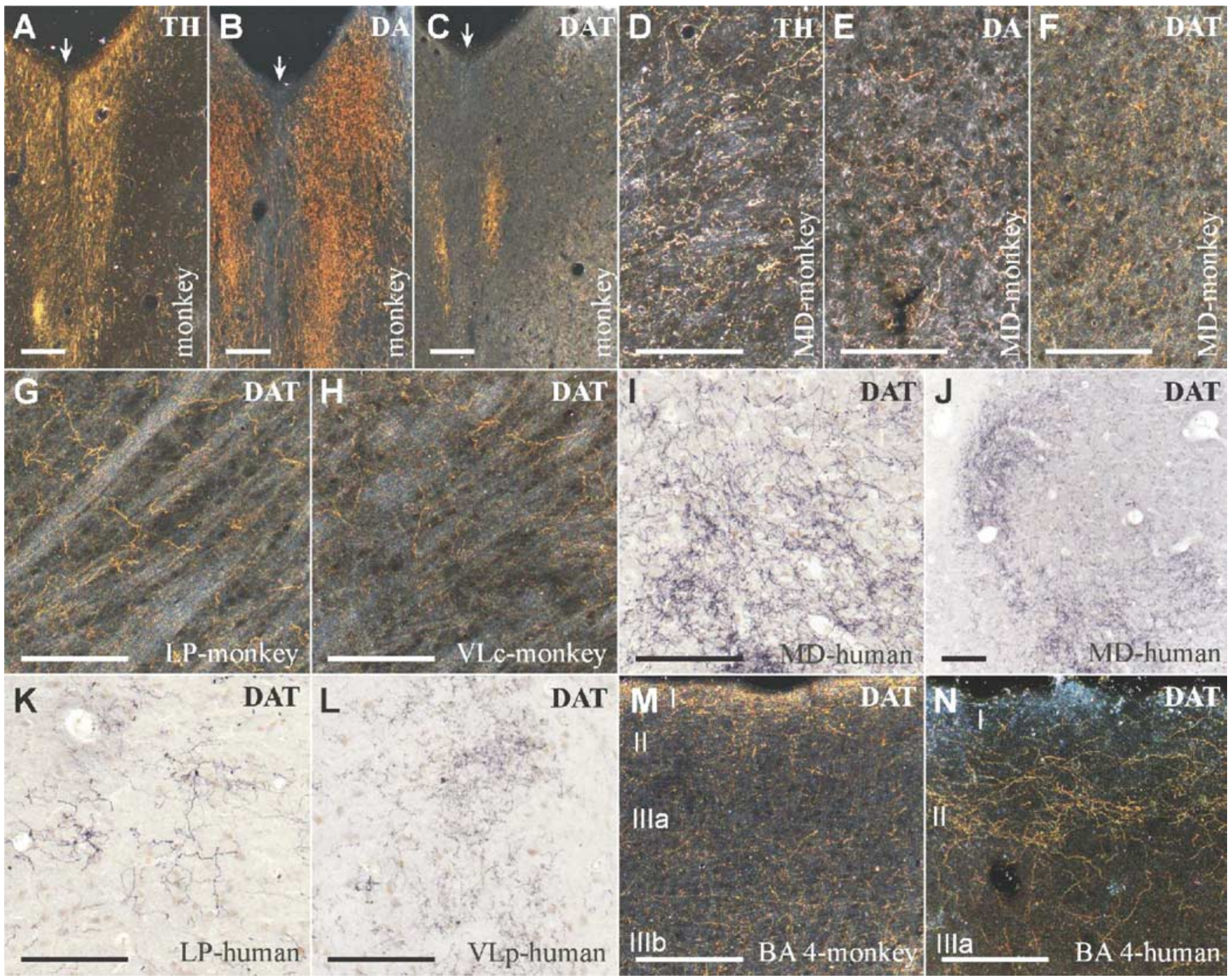

Figure 1. Dopaminergic axons profusely target the monkey and human thalamus. $\boldsymbol{A}-\boldsymbol{C}$, Axons immunoreactive for $\mathrm{TH}(\boldsymbol{A})$ and dopamine $(\boldsymbol{B})$ are densely concentrated in the midline thalamic region of the monkey (midline indicated by arrows), where DAT-ir axons $(\boldsymbol{C})$ are less abundant. $\boldsymbol{D}-\boldsymbol{N}$, Axons immunoreactive for TH $(\boldsymbol{D})$, dopamine $(\boldsymbol{E})$, and DAT $(\boldsymbol{F}-\boldsymbol{L})$ are abundant in the MD, LP, and VL nuclei of the monkey and human thalamus. Note that the density of the DAT-ir axons in the MD, LP, or VL nuclei of the monkey $(\boldsymbol{F}-\boldsymbol{H})$ and human $(\boldsymbol{I}-\boldsymbol{L})$ thalamus is similar to or even higher than that found in Brodmann's area 4 (BA 4) of the monkey $(\boldsymbol{M})$ and human $(\boldsymbol{N})$ cortex. Scale bars, $200 \mu \mathrm{m}$. DA, Dopamine; VLc, ventral lateral nucleus, caudal part; VLp, ventral lateral nucleus, posterior part.

cause TH reveals all dopaminergic neurons, including those that do not express DAT (see Table 1). By identifying the CTb-Au/D $\beta \mathrm{H}$ doublelabeled neurons at the transition between the mesencephalon and pons, it was possible to exclude the noradrenergic cell groups from the counts. At more rostral levels, including ventral mesencephalon, periaqueductal gray matter (PAG), and hypothalamus, $\mathrm{TH}$ reveals only dopaminergic neurons (Hökfelt et al., 1984).

Thalamic nomenclature. Names for the human thalamic nuclei followed the nomenclature by Hirai and Jones (1989); for the monkey thalamic nuclei, we used Olszewski's nomenclature (Olszewski, 1952) with some modifications (Cavada et al., 1995; Rico and Cavada, 1998).

\section{Results}

Dopaminergic axons profusely target the human and monkey thalamus

TH-ir axons were widely distributed in the human and monkey thalamus, showing their highest densities in midline nuclei (Fig. $1 A, D)$. TH immunoreaction, often used as a phenotypic marker for dopamine, may additionally reveal catecholamines other than dopamine (e.g., noradrenaline and adrenaline), because this synthetic enzyme is common to all catecholamines. This is why we additionally performed immunohistochemistry for dopamine in sections of the macaque monkey thalamus. The highest dopamine-ir axon density was observed in the midline limbic nuclei (Fig. $1 B$ ), followed by MD (Fig. $1 E$ ) and LP association nuclei and the VL motor nucleus. The lowest dopamine-ir axon density was in the intralaminar and the primary sensory relay nuclei.

To further confirm and characterize the dopamine input to the primate thalamus, we performed immunohistochemistry for DAT in adult human and macaque monkey brains. DAT reuptakes dopamine into the dopaminergic neuron and is a specific marker for the dopaminergic phenotype (Ciliax et al., 1999). The highly sensitive method used revealed a dense and widespread meshwork of DAT-ir axons that is distributed heterogeneously between and within the nuclei in both the human and monkey thalamus (Figs. $1 F-L, 2$ ). All the thalamic nuclei exhibit DAT-ir axons; the highest densities are found in the MD and LP association nuclei and the VL motor nucleus (Fig. 2). In the human thalamus, the VA motor nucleus also exhibits dense DAT-ir plex- 


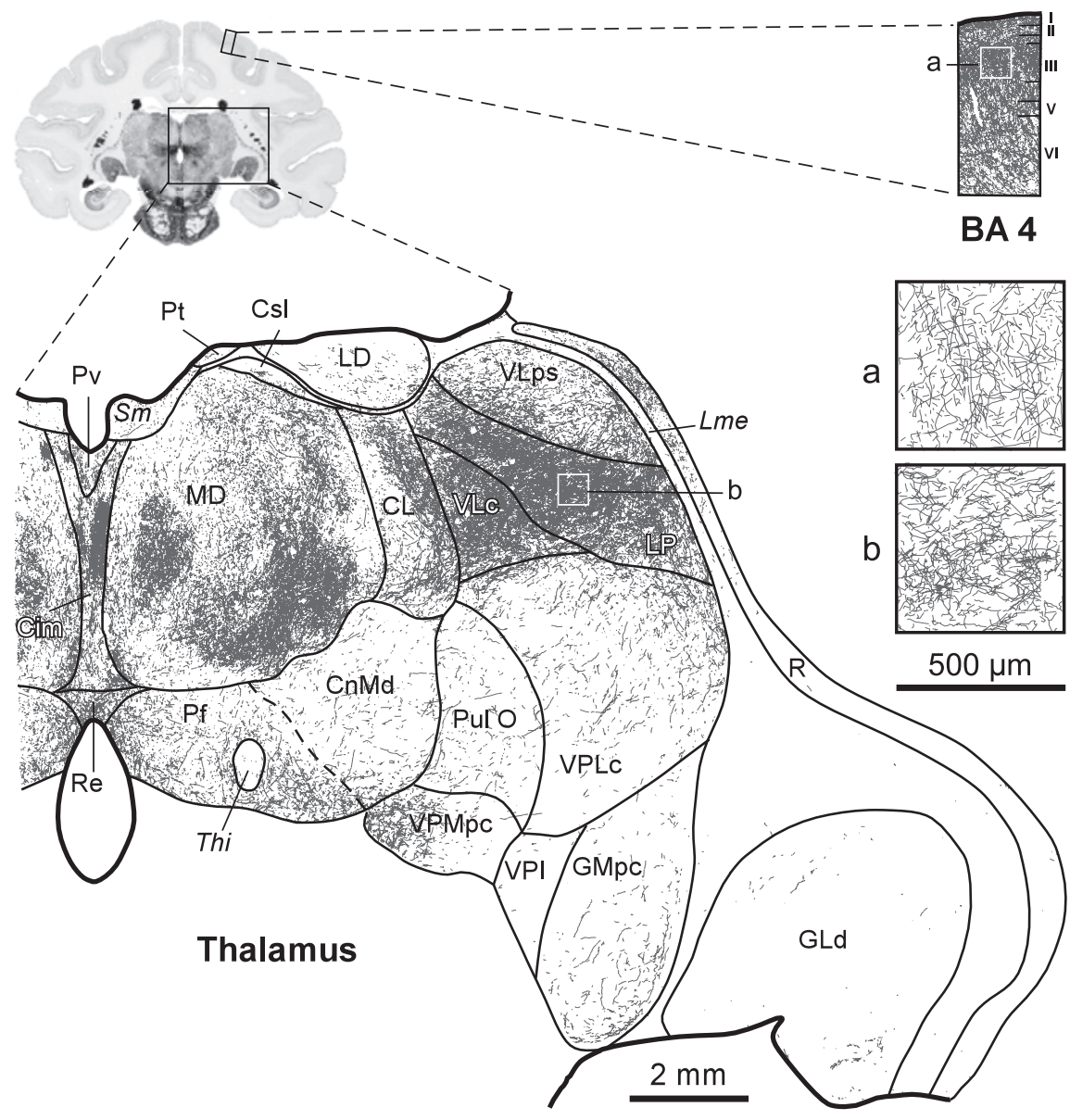

Figure 2. The DAT-ir axons have a widespread and uneven distribution in the macaque monkey thalamus. DAT-ir axons are present in every thalamic nuclei, reaching highest densities in MD, LP, and VLc. Compare the density of DAT-ir axons in these thalamic nuclei with the density in Brodmann's area 4 (BA 4) in the same section (both shown at the same magnification). Insets $\boldsymbol{a}$ and $\boldsymbol{b}$ show the cortical and thalamic regions framed on the squares at higher magnification, so that the lines from individual axon profiles are better visualized. Scale bars: drawings of the full hemithalamus and the cortical column, $2 \mathrm{~mm}$; insets $\boldsymbol{a}$ and $\boldsymbol{b}, 500$ $\mu \mathrm{m}$. Cim, Central nucleus, intermediate part; CL, central lateral nucleus; CnMd, centromedian nucleus; Csl, central nucleus, superior lateral part; LD, lateral dorsal nucleus; Lme, external medullary lamina; Pf, parafascicular nucleus; Pt, paratenial nucleus; Pul 0, oral pulvinar nucleus; Pv, paraventricular nucleus; R, reticular nucleus; Re, nucleus reuniens; Sm, stria medullaris; Thi, habenulo-interpeduncular tract; VLc, ventral lateral nucleus, caudal part; VLps, ventral lateral nucleus, pars postrema; VPI, ventral posterior inferior nucleus; VPMpc, ventral posterior medial nucleus, parvocellular part.

uses. The lowest densities are present in the primary sensory relay nuclei [dorsal lateral geniculate nucleus (GLd), visual; medial geniculate nucleus, parvocellular part $(\mathrm{GMpc})$, auditory; ventral posterior lateral nucleus, caudal part (VPLc), somatosensory] (Fig. 2). It is remarkable that, in both macaque and human, DAT-ir axon density in certain regions of MD, LP, and VL is as high as or higher than in the primary motor cortex, the cortical area with the densest dopamine innervation (Lewis et al., 2001) (Figs. 1, 2).

In contrast to the high density of $\mathrm{TH}$ - and dopamine-ir axons in the midline thalamic nuclei, DAT-ir axon density is only medium here, although there are some very dense patches (Figs. $1 A-C, 2)$. This discrepancy could be the result of a heterogeneous DAT content in the thalamic dopaminergic axons. Indeed, dopaminergic neurons express heterogeneous DAT levels (e.g., high levels in the substantia nigra vs undetectable levels in the hypothalamus) in all species reported to date, including humans and macaque monkeys (Table 1) (Cerruti et al., 1993; Ciliax et al., 1995, 1999; Haber et al., 1995). A different DAT expression among the dopaminergic neurons projecting to the midline and nonmidline thalamic nuclei would explain the differences in DAT-ir axonal density in the distinct thalamic regions.

\section{Multiple origin of thalamic dopamine} Unlike the dopaminergic systems identified to date (e.g., nigrostriatal, mesocortical, mesolimbic), the origin of the dopamine innervation in the primate thalamus is markedly diverse: all tracer injections in the macaque thalamus produced bilateral retrograde labeling in a wide range of dopaminergic neuronal populations (Table 1 , classification of dopaminergic groups). These included dopaminergic cell groups in the hypothalamus (A13-A15), PAG (A11), and ventral mesencephalon (A8A10), in addition to a group of putatively dopaminergic neurons in the lateral parabrachial nucleus ( $\mathrm{LPbN}) . \mathrm{CTb}-\mathrm{Au} / \mathrm{TH}$ double-labeled neurons were identified in all these dopaminergic cell groups (Figs. $3 I-M, 4)$. The injection in the midline thalamus produced the highest number of $\mathrm{CTb}-\mathrm{Au} / \mathrm{TH}$ double-labeled neurons (Fig. $3 M)$, consistent with the higher $\mathrm{TH}$-ir and dopamine-ir axon densities found in the midline. Likewise, the injection in VA, a nucleus with low $\mathrm{TH}$-ir, dopamine-ir, and DAT-ir axonal densities in the monkey, produced the lowest number of CTb$\mathrm{Au} / \mathrm{TH}$ double-labeled neurons (Fig. $3 M)$. Moreover, CTb-Au/DAT doublelabeled neurons were observed in dopaminergic groups whose neurons express DAT, including A8, A10, and A11 (Fig. $3 E-H)$.

The dopaminergic populations projected into the four injected thalamic regions with different intensities, and differences were especially apparent between the midline and nonmidline nuclei (Figs. $3 M, 4)$. The $\mathrm{A} 13$ dopaminergic group and the $\mathrm{LPbN}$ project intensely to the midline thalamus and have weaker projections to VA, MD, LP, and VL. Group A11 projects to all the injected regions, although the projection to $\mathrm{MD}$ was the strongest. Groups A10 and A8 project nearly equally throughout the examined thalamic nuclei. $\mathrm{CTb}-\mathrm{Au} / \mathrm{TH}$ neurons were infrequent in the substantia nigra compacta [group A9, dorsal tier (A9d) and ventral tier (A9v)] (Fig. 3M), except after the injection in LP and VL, from which the tracer spread minimally to the body of the caudate nucleus, and a group of labeled neurons was present in $\mathrm{A} 9 \mathrm{~d}$ and $\mathrm{A} 9 \mathrm{v}$. The absence of labeling in $\mathrm{A} 9 \mathrm{v}$ (origin of the nigrostriatal system) in all the other cases implies that, at least in the thalamic regions we have explored, the thalamic dopaminergic system is independent of the nigrostriatal system.

It is of note that the DAT content of the dopaminergic populations projecting to the thalamus is heterogeneous. Dopaminergic group A13 and the LPbN, which project heavily to the midline thalamus, are virtually devoid of DAT (Table 1) (Ciliax et al., 1999). This explains the lower density of DAT-ir axons in the midline thalamic nuclei relative to $\mathrm{TH}$-ir or dopamine-ir axons (Figs. 1A-C, 2). In contrast, the dopaminergic neurons of groups 
A8-A11 exhibit variable amounts of DAT (Table 1) (Haber et al., 1995; Ciliax et al., 1999) and have a larger relative weight in the dopamine innervation of the thalamic nuclei with prominent densities of DAT-ir axons (MD, LP, and VL) (Figs. 3M, 4).

\section{Discussion}

This study provides the first demonstration of widespread and profuse innervation of the primate thalamus by dopamine. We show that the dopamine innervation of the human and macaque thalamus has a complex organization in terms of distribution, density, DAT content, and origin. The most densely innervated thalamic regions are the limbic midline nuclei, the higher-order MD and LP, and the motor VL nucleus. In the midline nuclei, dopaminergic axons that do not express DAT are more prevalent than those that express DAT. The latter are more prevalent in nonmidline thalamic nuclei. The dopamine innervation of the primate thalamus originates bilaterally in multiple dopaminergic neuronal populations of the hypothalamus, PAG, ventral mesencephalon, and the putatively dopaminergic neurons of the $\mathrm{LPbN}$. The multiple origin of thalamic dopamine contrasts with that of the established nigrostriatal, mesocortical, and mesolimbic dopaminergic systems, the neurons of origin of which are restricted to the mesencephalon (Lewis and Sesack, 1997).

The present report of prominent dopamine innervation in the primate thalamus is based on the following two lines of evidence: (1) immunodetection in the thalamus of three dopamine markers (TH, dopamine, and DAT, a specific marker of the dopaminergic phenotype) and (2) identification of dopaminergic neurons projecting into the thalamus by retrograde tract-tracing combined with immunohistochemistry. The distributions and relative densities of labeled fibers in the thalamus obtained with the three antibodies were similar overall. A mismatch was present in the midline, where DAT-ir axons were less dense than TH-ir and dopamine-ir axons (Fig. $1 A-C$ ). This seeming discrepancy is explained by the fact that the majority of dopaminergic afferents to the midline originate in DAT-negative dopaminergic groups of the hypothalamus and the putatively dopaminergic neurons of LPbN (Figs. 3M, 4).

The larger extent of the dopamine innervation in the primate thalamus compared with that in the rodent (Groenewegen, 1988; Papadopoulos and Parnavelas, 1990) appears to be an evolutionary trend that also occurs with the dopamine innervation of the cerebral cortex (Berger et al., 1991). The increase in dopaminoceptive areas in the thalamus and cortex could be related to the specific higher brain capabilities of primates. In fact, three of the most densely dopamine-innervated thalamic nuclei (midline, MD, LP) are specifically connected with higher association frontal and parietal cortical regions.

Although widespread dopamine innervation in the primate thalamus has never been reported previously, our findings concur with diverse data in the literature. The presence of dopamine in the thalamus was reported in human and monkey brains using biochemical tools (Brown et al., 1979; Goldman-Rakic and Brown, 1981). More recently, DAT has been detected by immu- nohistochemistry in the monkey MD nucleus (Melchitzky and Lewis, 2001) and by in vivo binding of radioligands in the human thalamus (Wang et al., 1995). It is interesting that the thalamus has been shown to hold higher concentrations of dopamine and more intense DAT binding than the cerebral cortex (Brown et al., 1979; Goldman-Rakic and Brown, 1981; Wang et al., 1995), observations that fit with the present data of similar or higher densities of dopaminergic axons in some thalamic territories than in cortical area 4 (Fig. 2). Moreover, $\mathrm{D}_{2}$-like dopamine receptor binding sites have been shown in the human thalamus using in vivo radioligands, with a distribution that resembles that of the dopamine innervation described here (Rieck et al., 2004). Finally, dopamine and $\mathrm{D}_{1}$-like and $\mathrm{D}_{2}$-like dopamine receptor agonists modulate visual responses of the cat dorsal lateral geniculate thalamic nucleus (Zhao et al., 2001, 2002).

An elevated dopamine content has been reported in the thalamus of schizophrenic patients (Oke and Adams, 1987), and in vivo binding has demonstrated low levels of $\mathrm{D}_{2} / \mathrm{D}_{3}$-like dopamine receptors in the thalamus of nonmedicated schizophrenics (Talvik et al., 2003; Yasuno et al., 2004). Moreover, reduced thalamic volume (Andreasen et al., 1994; Buchsbaum et al., 1996) and activity (Buchsbaum et al., 1996), as well as specific activation of the thalamus during hallucinations (Silbersweig et al., 1995), have been reported in schizophrenic patients. Despite these reports, the notion of a thalamic dopaminergic alteration in schizophrenia has not gained much attention, probably because the existence of significant dopamine innervation in the human thalamus was unsuspected. The dopamine innervation of the thalamus that we demonstrate is especially dense in thalamic regions strongly connected with the cortical areas most profoundly affected in schizophrenia (prefrontal and limbic cortices, respectively connected with MD and midline thalamic nuclei).

Regarding DAT expression, two broad "dopaminergic domains" can be identified in the thalamus: a midline domain, poor in DAT, with its dopamine axons preferentially originating in hypothalamic groups (especially A13) and the LPbN; and a nonmidline domain, richer in DAT, the neurons of origin of which 


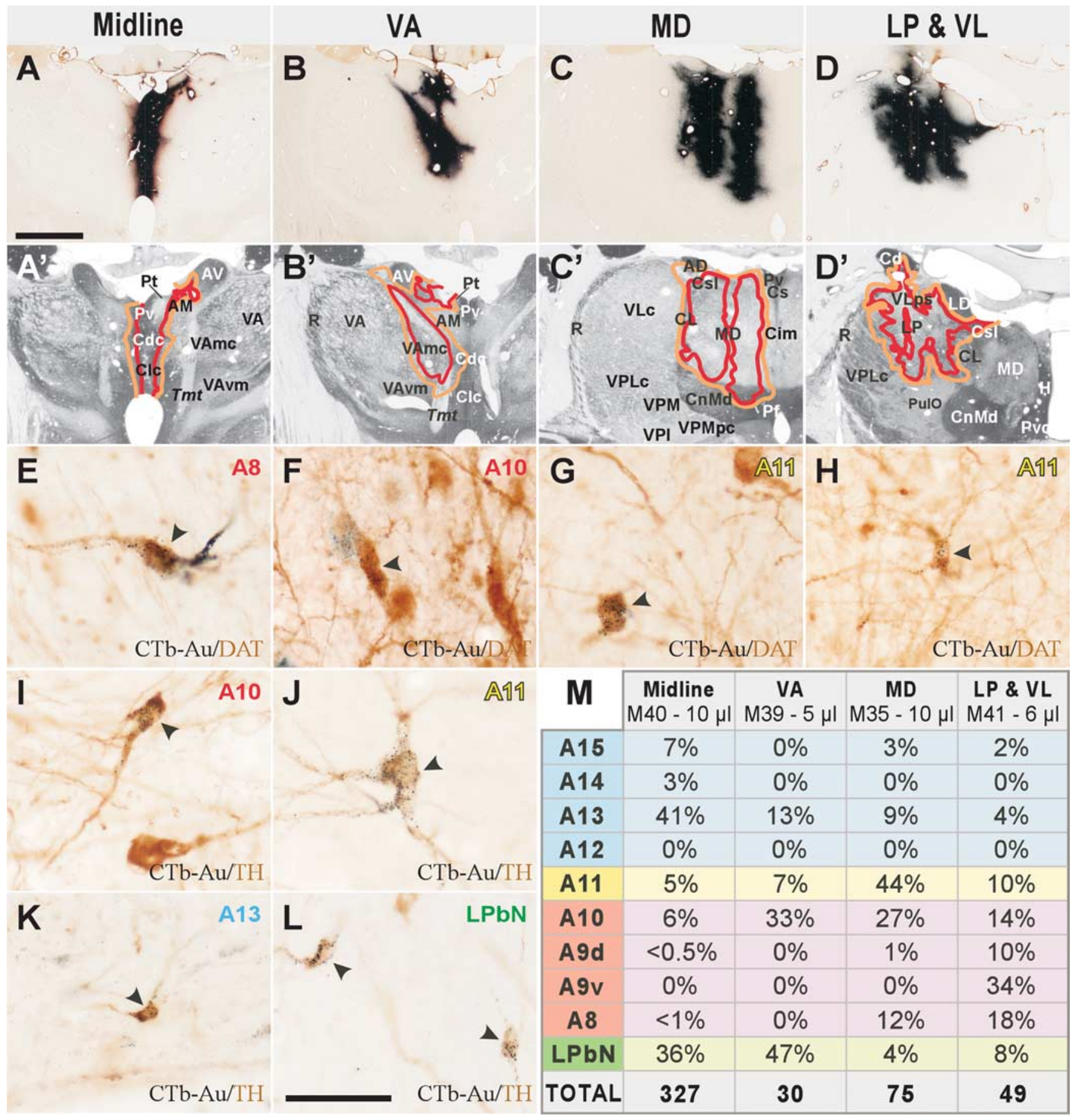

Figure 3. The dopamine innervation of the macaque thalamus originates in multiple dopaminergic populations. $\boldsymbol{A}-\boldsymbol{D}^{\prime},(\mathbf{C}$-Au injection sites in the thalamus $(\boldsymbol{A}-\boldsymbol{D})$ and schematic representations of the targeted thalamic nuclei drawn over adjacent sections processed for acetylcholinesterase $\left(\boldsymbol{A}^{\prime}-\boldsymbol{D}^{\prime}\right)$. The injected thalamic regions are the midline nuclei $\left(\boldsymbol{A}, \boldsymbol{A}^{\prime}\right), \mathrm{VAmc}\left(\boldsymbol{B}, \boldsymbol{B}^{\prime}\right), \mathrm{MD}(\boldsymbol{C}$, $\left.\boldsymbol{C}^{\prime}\right)$ and $L P, V L p s$, and VLc (VLcin a level more rostral to that shown) $\left(\boldsymbol{D}, \boldsymbol{D}^{\prime}\right) . \boldsymbol{E}-\boldsymbol{L}, \mathrm{CTb}-\mathrm{Au} / \mathrm{DAT}(\boldsymbol{E}-\boldsymbol{H})$ and $(\mathrm{Tb}-\mathrm{Au} / \mathrm{TH}(\boldsymbol{I}-\boldsymbol{L})$ double-labeled neurons (indicated by arrowheads) in some dopaminergic groups. Black dots in $\boldsymbol{E}-\boldsymbol{L}$ denote $\mathrm{CTb}$-Au; the diffuse brown staining is the reaction product of the DAT or TH immunoreaction. $\boldsymbol{M}$, Table showing the percentages of CTb-Au/TH double-labeled neurons in each dopaminergic group for each injection, as well as the total number of double-labeled neurons in 1/10 of the sections (color coding is the same as in Table 1 and Fig. 4). Scale bars: (in A) $\boldsymbol{A}-\boldsymbol{D}^{\prime}, 3 \mathrm{~mm}$; (in $\left.\boldsymbol{L}\right) \boldsymbol{E}-\boldsymbol{L}, 50 \mu \mathrm{m}$. AM, Anteromedial nucleus; AV, anteroventral nucleus; $C$ d, caudate nucleus; $C \mathrm{Cd}$, central nucleus, densocellular part; Cim, central nucleus, intermediate part; $\mathrm{CL}$, central lateral nucleus; Clc, central nucleus, latocellular part; CnMd, centromedian nucleus; $C$, central nucleus, superior part; Csl, central nucleus, superior lateral part; LD, lateral dorsal nucleus; Pt, paratenial nucleus; Pul0, oral pulvinar nucleus; Pv, paraventricular nucleus; R, reticular nucleus; Tmt, mammillothalamic tract; VAmc, ventral anterior nucleus, magnocellular part; VAvm, ventral anterior nucleus, ventromedial part; VLc, ventral lateral nucleus, caudal part; VLps, ventral lateral nucleus, pars postrema; VPI, ventral posterior inferior nucleus; VPM, ventral posterior medial nucleus; VPMpc, ventral posterior medial nucleus, parvocellular part; AD, anterodorsal nucleus; $\mathrm{H}$, habenular nuclei; Pf, parafascicular nucleus; Pvc, paraventricular nucleus, caudal part.

preferentially lie in the A8-A11 dopaminergic groups. Because DAT controls the time that dopamine remains in the extracellular space and the distance it reaches from its point of release (Cragg and Rice, 2004), dopamine signaling must be more spatially and temporally restricted in the nonmidline domain than in the midline domain. Moreover, the heterogeneity of DAT content in the primate thalamus could mean that the different thalamic dopaminergic circuits would respond differently to neurodegenera- 


\section{Thalamus}

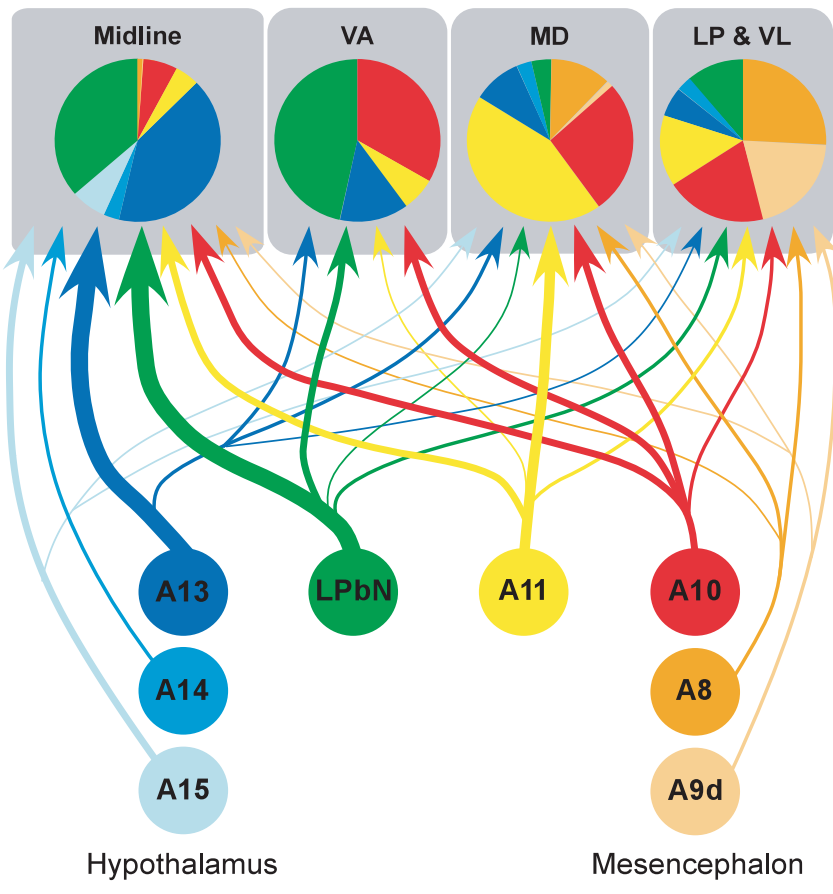

Figure 4. Summary diagram of the dopamine projection to the macaque monkey thalamus. The various dopaminergic groups that innervate the thalamus (represented as colored circles; color coding is the same as in Table 1) heterogeneously project to the four studied thalamic regions. The relative contribution of each group to the innervation of each thalamic area is color coded in the pie charts. Line thickness is correlated to the intensity of the projection.

tive or addictive processes. Interestingly, DAT is the cellular entry point for toxins that selectively destroy dopaminergic neurons, such as 1-methyl-4-phenylpyridinium and 6-OHDA (Pifl and Caron, 2002). Stimulant drugs such as cocaine or amphetamine exert their actions by binding to DAT and increasing the extracellular concentration of dopamine: cocaine blocks dopamine reuptake, and amphetamine induces dopamine efflux from inside the dopaminergic neuron (Pifl and Caron, 2002; Kahlig et al., 2005). Thus, the DAT-positive axons of the thalamus, where binding of labeled cocaine and methylphenidate (an amphetamine derivative) has been demonstrated (Wang et al., 1995), are potential sites of action for the above drugs.

The widespread origin of thalamic dopamine from functionally and chemically diverse dopaminergic groups (Figs. 3, 4) may confer unique functional properties on this system. Dopaminergic neurons of the ventral mesencephalon (groups A8-A10) code for the presence of reward-related stimuli (Schultz, 2002; Fiorillo et al., 2003). The stimuli that effectively trigger other dopaminergic neuronal groups are not known. However, PAG is reported to be a nodal point in the reaction to aversive stimuli (Bandler et al., 2000), and the dopaminergic neurons of the hypothalamus (groups A12-A15) and LPbN are likely to be involved in coding aspects related to the internal state of the individual (Tillet, 1994; Johnson and Thunhorst, 1997). Dopamine could thus modulate thalamic relay both in relationship to reward/endangering features of environmental stimuli and to visceral/hormonal signals from the internal milieu.

In sum, the primate thalamus is endowed with a robust and complex dopamine innervation that in all likelihood influences the activity of the cortical, striatal, and amygdaloid regions to which the thalamus is connected. The dopaminergic system of the primate thalamus described here merits additional study to unravel its functional significance under normal and pathological conditions such as schizophrenia, Parkinson's disease, and drug addiction.

\section{References}

Agid Y (1991) Parkinson's disease: pathophysiology. Lancet 337:1321-1324.

Alonso JR, Amaral DG (1995) Cholinergic innervation of the primate hippocampal formation. I. Distribution of choline acetyltransferase immunoreactivity in the Macaca fascicularis and Macaca mulatta monkeys. J Comp Neurol 355:135-170.

Andreasen NC, Arndt S, Swayze VI, Cizadlo T, Flaum M, O'Leary D, Ehrhardt JC, Yuh WTC (1994) Thalamic abnormalities in schizophrenia visualized through magnetic resonance image averaging. Science 266:294-298.

Aosaki T, Graybiel AM, Kimura M (1994) Effect of the nigrostriatal dopamine system on acquired neural responses in the striatum of behaving monkeys. Science 265:412-415.

Arsenault M-Y, Parent A, Séguéla P, Descarries L (1988) Distribution and morphological characteristics of dopamine-immunoreactive neurons in the midbrain of the squirrel monkey (Samiri sciureus). J Comp Neurol 267:489-506.

Bandler R, Keay KA, Floyd N, Price J (2000) Central circuits mediating patterned autonomic activity during active vs. passive emotional coping. Brain Res Bull 53:95-104.

Berger B, Gaspar P, Verney C (1991) Dopaminergic innervation of the cerebral cortex: unexpected differences between rodents and primates. Trends Neurosci 14:21-27.

Bissiere S, Humeau Y, Luthi A (2003) Dopamine gates LTP induction in lateral amygdala by suppressing feedforward inhibition. Nat Neurosci 6:587-592.

Björklund A, Nobin A (1973) Fluorescence histochemical and microspectrofluorometric mapping of dopamine and noradrenaline cell groups in the rat diencephalon. Brain Res 51:193-205.

Brown RM, Crane AM, Goldman PS (1979) Regional distribution of monoamines in the cerebral cortex and subcortical structures of the rhesus monkey: concentrations and in vivo synthesis rates. Brain Res 168:133-150.

Brozoski TJ, Brown RM, Rosvold HE, Goldman PS (1979) Cognitive deficits caused by regional depletion of dopamine in prefrontal cortex of rhesus monkey. Science 205:929-932.

Buchsbaum MS, Someya T, Teng CY, Abel L, Chin S, Najafi A, Haier RJ, Wu J, Bunney Jr WE (1996) PET and MRI of the thalamus in nevermedicated patients with schizophrenia. Am J Psychiatry 153:191-199.

Cavada C, Compañy T, Hernández-González A, Reinoso-Suárez F (1995) Acetylcholinesterase histochemistry in the macaque thalamus reveals territories selectively connected to frontal, parietal and temporal association cortices. J Chem Neuroanat 8:245-257.

Cerruti C, Walther DM, Kuhar MJ, Uhl GR (1993) Dopamine transporter mRNA expression is intense in rat midbrain neurons and modest outside midbrain. Mol Brain Res 18:181-186.

Ciliax BJ, Heilman C, Demchyshyn LL, Pristupa ZB, Ince E, Hersch SM, Niznik HB, Levey AI (1995) The dopamine transporter: immunochemical characterization and localization in brain. J Neurosci 15:1714-1723.

Ciliax BJ, Drash GW, Staley JK, Haber S, Mobley CJ, Miller GW, Mufson EJ, Mash DC, Levey AI (1999) Immunocytochemical localization of the dopamine transporter in human brain. J Comp Neurol 409:38-56.

Cragg SJ, Rice ME (2004) DAncing past the DAT at a DA synapse. Trends Neurosci 27:270-277.

Dahlström A, Fuxe K (1964) Evidence for the existence of monoaminecontaining neurons in the central nervous system. I. Demonstration of monoamines in the cell bodies of brain stem neurons. Acta Physiol Scand 62 [Suppl 232]:1-55.

Fiorillo CD, Tobler PN, Schultz W (2003) Discrete coding of reward probability and uncertainty by dopamine neurons. Science 299:1898-1902.

Gallyas F (1979) Silver staining of myelin by means of physical development. Neurol Res 1:203-209.

Goldman-Rakic P, Brown RM (1981) Regional changes of monoamines in cerebral cortex and subcortical structures of aging rhesus monkeys. Neuroscience 6:177-187.

Groenewegen HJ (1988) Organization of the afferent connections of the mediodorsal thalamic nucleus in the rat, related to the mediodorsalprefrontal topography. Neuroscience 24:379-431.

Guillery RW, Sherman SM (2002) Thalamic relay functions and their role in 
corticocortical communication: generalizations from the visual system. Neuron 33:163-175.

Haber SN, Ryoo H, Cox C, Lu W (1995) Subsets of midbrain dopaminergic neurons in monkeys are distinguished by different levels of mRNA for the dopamine transporter: comparison with the mRNA for the D2 receptor, tyrosine hydroxylase and calbinding immunoreactivity. J Comp Neurol 362:400-410.

Hirai T, Jones EG (1989) A new parcellation of the human thalamus on the basis of histochemical staining. Brain Res Brain Res Rev 14:1-34.

Hökfelt T, Martensson R, Björklund A, Kleinau S, Goldstein M (1984) Distributional maps of tyrosine-hydroxylase-immunoreactive neurons in the rat brain. In: Handbook of chemical neuroanatomy, Vol 2, Classical transmitters in the CNS, Part I (Björklund A, Hökfelt T, eds), pp 277-379. Amsterdam: Elsevier.

Johnson AK, Thunhorst RL (1997) The neuroendocrinology of thirst and salt appetite: visceral sensory signals and mechanisms of central integration. Front Neuroendocrinol 18:292-353.

Kahlig KM, Binda F, Khoshbouei H, Blakely RD, McMahon DG, Javitch JA, Galli A (2005) Amphetamine induces dopamine efflux through a dopamine transporter channel. Proc Natl Acad Sci USA:3495-3500.

Kitahama K, Ikemoto K, Jouvet A, Nagatsu I, Sakamoto N, Pearson J (1998) Aromatic L-amino acid decarboxyase- and tyrosine hydroxylaseimmunohistochemistry in the adult human hypothalamus. J Chem Neuroanat 16:43-55.

Lewis DA, Sesack SR (1997) Dopamine systems in the primate brain. In: The primate nervous system, Part I (Bloom FE, Björklund A, Hökfelt T, eds), pp 263-375. Amsterdam: Elsevier.

Lewis DA, Melchitzky DS, Sesack SR, Whitehead RE, Auh S, Sampson A (2001) Dopamine transporter immunoreactivity in monkey cerebral cortex: regional, laminar, and ultrastructural localization. J Comp Neurol 432:119-136

McCormick DA (1992) Neurotransmitter actions in the thalamus and cerebral cortex and their role in neuromodulation of thalamocortical activity. Prog Neurobiol 39:337-388.

Melchitzky DS, Lewis DA (2001) Dopamine transporter-immunoreactive axons in the mediodorsal thalamic nucleus of the macaque monkey. Neuroscience 103:1033-1042.

Nicola SM, Surmeier J, Malenka RC (2000) Dopaminergic modulation of neuronal excitability in the striatum and nucleus accumbens. Annu Rev Neurosci 23:185-215.

Oke AF, Adams RN (1987) Elevated thalamic dopamine: possible link to sensory dysfunctions in schizophrenia. Schizophr Bull 13:589-604.

Olszewski J (1952) The thalamus of the Macaca mulatta. An atlas for use with the sterotaxic instrument. Basel, Switzerland: Karger

Papadopoulos GC, Parnavelas JG (1990) Distribution and synaptic organization of dopaminergic axons in the lateral geniculate nucleus of the rat. J Comp Neurol 294:356-361.

Pifl C, Caron M (2002) The dopamine transporter: molecular biology, pharmacology and genetics. In: Dopamine in the CNS I (Borrelli E, Di Chiara G, Wang S, eds), pp 257-298. New York: Springer.
Rico B, Cavada C (1998) Adrenergic innervation of the monkey thalamus: an immunohistochemical study. Neuroscience 84:839-847.

Rieck RW, Ansari MS, Whetsell Jr WO, Deutch AY, Kessler RM (2004) Distribution of dopamine D2-like receptors in the human thalamus: autoradiographic and PET studies. Neuropsychopharmacology 29:362-372.

Rub U, Del Tredici K, Schultz C, Ghebremedhin E, de Vos RA, Jansen Steur E, Braak H (2002) Parkinson's disease: the thalamic components of the limbic loop are severely impaired by alpha-synuclein immunopositive inclusion body pathology. Neurobiol Aging 23:245-254.

Schultz W (2002) Getting formal with dopamine and reward. Neuron $36: 241-263$

Sherman SM, Guillery RW (1996) Functional organization of thalamocortical relays. J Neurophysiol 76:1367-1395.

Sherman SM, Guillery RW (2004) Thalamus. In: The synaptic organization of the brain, Ed 5 (Shepherd GM, ed), pp 311-359. New York: Oxford UP.

Shu SY, Ju G, Fan LZ (1988) The glucose oxidase-DAB-nickel method in peroxidase histochemistry of the nervous system. Neurosci Lett 85:169-171.

Silbersweig DA, Stern E, Frith C, Cahill C, Holmes A, Grootoonk S, Seaward J, McKenna P, Chua SE, Schnorr L, Jones T, Frackoviak RSJ (1995) A functional neuroanatomy of hallucinations in schizophrenia. Nature 378:176-179.

Steriade M, Jones EG, McCormick DA (1997) Thalamus. Amsterdam: Elsevier Science.

Talairach J, Tournoux P (1988) Co-planar stereotaxic atlas of the human brain. 3-dimensional proportional system: an approach to cerebral imaging. Stuttgart, Germany: Thieme.

Talvik M, Nordstrom AL, Olsson H, Halldin C, Farde L (2003) Decreased thalamic D2/D3 receptor binding in drug-naive patients with schizophrenia: a PET study with [11C]FLB 457. Int J Neuropsychopharmacol 6:361-370.

Tillet Y (1994) Catecholaminergic neuronal systems in the diencephalon of mammals. In: Phylogeny and development of catecholamine systems in the CNS of vertebrates (Smeets WJA, Reiner A, eds), pp 207-246. Cambridge: Cambridge UP.

Wang GJ, Volkow ND, Fowler JS, Ding YS, Logan J, Gatley SJ, MacGregor RR, Wolf AP (1995) Comparison of two PET radioligands for imaging extrastriatal dopamine transporters in human brain. Life Sci 57:PL187-PL191.

Yasuno F, Suhara T, Okubo Y, Sudo Y, Inoue M, Ichimiya T, Takano A, Nakayama K, Halldin C, Farde L (2004) Low dopamine d(2) receptor binding in subregions of the thalamus in schizophrenia. Am J Psychiatry 161:1016-1022.

Zhao Y, Kerscher N, Eysel U, Funke K (2001) Changes of contrast gain in cat dorsal lateral geniculate nucleus by dopamine receptor agonists. NeuroReport 12:2939-2945.

Zhao Y, Kerscher N, Eysel U, Funke K (2002) D1 and D2 receptor-mediated dopaminergic modulation of visual responses in cat dorsal lateral geniculate nucleus. J Physiol (Lond) 539:223-238. 